\title{
Acknowledgment of Reviewers, 2012
}

The success of Phytopathology depends on the quality of manuscripts submitted by authors and on the care and competence with which they are reviewed. It is the policy of the Editorial Board to solicit reviews for manuscripts from specialists who are most qualified to review them. In addition to members of the Editorial Board, the individuals listed below provide constructive critical reviews of one or more manuscripts during the past year. Their names are published here in grateful appreciation of their contribution to the journal and to the science of phytopathology. http://dx.doi.org/10.1094/PHYTO-103-1-0004

Able, A. J., University of Adelaide, Glen Osmond, South Australia, Australia

Adaskaveg, J. E., University of California, Riverside, CA, U.S.A.

Adhikari, T., North Dakota State University, Fargo, ND, U.S.A.

Agudelo, P., Clemson University, Clemson, SC, U.S.A.

Akimitsu, K., Kagawa University, Miki, Kagawa, Japan

Aldwinckle, H., Cornell University, Geneva, NY, U.S.A.

Allen, T. W., Mississippi State University, Stoneville, MS, U.S.A.

Almeida, R., University of California, Berkeley, CA, U.S.A.

Alvarado, V., Texas A\&M University, College Station, TX, U.S.A.

Alvarez, A., University of Hawaii, Honolulu, HI, U.S.A.

Amorim, L., Universidade de São Paulo, Piracicaba, São Paulo,

Brazil

Angelini, R., University, Rome, Italy

Ashikawa, I., NARO Institute of Crop Science, Tsukuba, Ibaraki,

Japan

Atallah, Z., Hartnell College, Salinas, CA, U.S.A.

Avenot, H. F., University of California Davis, Parlier, CA, U.S.A.

Bailey, A. M., University of Bristol, Bristol, United Kingdom

Barak, J., University of Wisconsin, Madison, WI, U.S.A.

Battilani, P., Università Cattolica del Sacro Cuore, Piacenza, Italy

Beattie, A. D., University of Saskatchewan, Saskatoon, Saskatchewan,

Canada

Beaulieu, C., Université de Sherbrooke, Sherbrooke, Quebec, Canada

Ben-David, R., University of Zurich, Zurich, Switzerland

Bockus, W., Kansas State University, Manhattan, KS, U.S.A.

Bonants, P., Plant research International, Wageningen, the

Netherlands

Bostock, R. M., University of California, Davis, CA, U.S.A.

Bradeen, J., St. Paul, MN, U.S.A.

Brenneman, T., University of Georgia, Tifton, GA, U.S.A.

Briddon, R. W., National Institute for Biotechnology and Genetic

Engineering, Faisalabad, Pakistan

Brlansky, R. H., University of Florida, Lake Alfred, FL, U.S.A.

Broders, K., University of New Hampshire, Durham, NH, U.S.A.

Brown, R. L., USDA-ARS-SRRC, New Orleans, LA, U.S.A.

Brueggeman, R. S., North Dakota State University, Fargo, ND,

U.S.A.

Bull, C. T., USDA-ARS, Salinas, CA, U.S.A.

Burdman, S., The Hebrew University of Jerusalem, Rehovot, Israel

Byrne, H. D., Texas A\&M University, College Station, TX, U.S.A.

Caffi, T., Università Cattolica del Sacro Cuore, Piacenza, Italy

Cardinal, A., North Carolina State University, Raleigh, NC, U.S.A.

Carter, L., Syngenta Seeds, Inc., Nampa, ID, U.S.A.

Carter, M., USDA-ARS, Albany, CA, U.S.A.

Caruso, F.

Castillo, P., Institute for Sustainable Agriculture, Cordoba, Spain

Castlebury, L., Beltsville, U.S.A.

Catara, V., University of Catania, Catania, Italy

Cazorla, F. M., Málaga, Spain

Chang, J., Oregon State University, Corvallis, OR, U.S.A.

Charkowski, A. O., University of Wisconsin, Madison, WI, U.S.A.

Chastagner, G. A., Washington State University, Puyallup, WA,

U.S.A.

Chaverri, P., University of Maryland, College Park, MD, U.S.A.

Chellemi, D. O., U.S. Department of Agriculture, Fort Pierce, FL,

U.S.A.

Chen, J., USDA-ARS, Parlier, CA, U.S.A.
Chen, X., USDA-ARS and Washington State University, Pullman, WA, U.S.A.

Chen, Z., Louisiana State University Agricultural Center, Baton Rouge, LA, U.S.A.

Chilvers, M. I., Michigan State University, East Lansing, MI, U.S.A.

Chung, C., National Taiwan University, Taipei City, Taiwan

Chung, K., University of Florida, Lake Alfred, FL, U.S.A.

Civerolo, E. L., USDA, Parlier, CA, U.S.A.

Cobine, $\mathrm{P}$.

Cohen, Y. R., Bar-Ilan University, Ramat-Gan, Israel

Cook, R. T., York, U.S.A.

Cooke, D. E., Scottish Crop Research Institute, Dundee, United Kingdom

Cooksey, D., University of California, Riverside, CA, U.S.A.

Correll, J. C., University of Arkansas, Fayetteville, AR, U.S.A.

Cottyn, B., Institute for Agricultural and Fisheries Research (ILVO),

Merelbeke, Belgium

Cox, K. D., Cornell University, Geneva, NY, U.S.A.

Cunniffe, N. J., University of Cambridge, Cambridge, United Kingdom

Damann, K. E., Louisiana State University Agricultural Center,

Baton Rouge, LA, U.S.A.

Datnoff, L. E., Louisiana State University, Baton Rouge, LA, U.S.A.

Davis, M., University of California, Davis, CA, U.S.A.

Day, B., Michigan State University, East Lansing, MI, U.S.A.

de la Fuente, L., Auburn University, Auburn, AL, U.S.A.

De Wolf, E., KSU, Manhattan, KS, U.S.A.

del Carmen, M., Consejo Superior de Investigaciones Científicas

(Misión Biológica de Galicia), Pontevedra, Pontevedra, Spain

Del Ponte, E., Universidade Federal do Rio Grande do Sul, Porto Alegre, Rio Grande do Sul, Brazil

del Rio, L., ND State University, Fargo, ND, U.S.A.

Delmotte, F., INRA, Villenave d'Ornon Cedex, France

den Nijs, L., Plant Protection Service, Wageningen, Netherlands

Denny, T., University of Georgia, Athens, GA, U.S.A.

Dewdney, M. M., University of Florida, Lake Alfred, FL, U.S.A.

Dhawan, R., Monsanto, Chesterfield, MO, U.S.A.

Dobinson, K., Agriculture \& Agri-Food Canada, London, Ontario, Canada

Dong, H., Nanjing Agricultural University, Nanjing, China

Duffy, B., Agroscope Changins-Wädenswil, Wädenswil, Switzerland

Dumenyo, K., Tennessee State University, Nashville, TN, U.S.A.

Dutech, C., INRA, Cestas, U.S.A.

Ebbole, D., Texas A\&M University, College Station, TX, U.S.A.

Edwards, M., USDA-ARS, Fargo, ND, U.S.A.

Eid, S., Moscow, ID, U.S.A.

Elad, Y., Volcani Center, Bet Dagan, Israel

Elling, A., U.S.A.

Ellis, M. L., The Ohio State University, Wooster, OH, U.S.A.

Erickson, $\mathrm{M}$.

Esker, P., Universidad de Costa Rica, San Jose, Costa Rica

Eskes, B., CIRAD, Montpellier, France

Farman, M., University of Kentucky, Lexington, KY, U.S.A.

Fernandes, M., Embrapa, Passo Fundo, RS, Brazil

Ferrandino, F. J., The Connecticut Agricultural Experiment Station, New Haven, CT, U.S.A.

Fountaine, J.

Fraaije, B., Rothamsted Research, Harpenden, Hertfordshire, United Kingdom 
Franc, G.

Franz, E.

French, R., Texas AgriLife Extension Service, Amarillo, TX, U.S.A. French, R., USDA-ARS, Lincoln, NE, U.S.A.

Friesen, T., Northern Crop Science Laboratory, Fargo, ND, U.S.A.

Fry, W., Cornell University, Ithaca, NY, U.S.A.

Gadoury, D., Cornell University, NYSAES, Geneva, NY, U.S.A.

Gale, L. R., University of Minnesota, St. Paul, MN, U.S.A.

Garbelotto, M., University of California, Berkeley, CA, U.S.A.

Garvin, D., USDA-ARS, St. Paul, MN, U.S.A.

Gasparich, G., Towson University, Towson, MD, U.S.A.

Gent, D. H., USDA-ARS, Corvallis, CO, U.S.A.

Gevens, A. J., University of Wisconsin, Madison, WI, U.S.A.

Ghosh, A., Kansas State University, Manhattan, KS, U.S.A.

Giblin Davis, R., University of Florida-IFAS, Davie, FL, U.S.A.

Gibson, G., Heriot Watt University, Edinburgh, United Kingdom

Gilbertson, R., University of California, Davis, CA, U.S.A.

Gold, S., USDA-ARS, Athens, GA, U.S.A.

Goodin, M.

Goodwin, S., USDA-ARS, West Lafayette, IN, U.S.A.

Gordon, T., University of California, Davis, CA, U.S.A.

Gosme, M., INRA, Le Rheu cedex, France

Goss, E. M., University of Florida, Gainesville, FL, U.S.A.

Gottwald, T., U.S. Department of Agriculture, Ft. Pierce, FL, U.S.A.

Graham, J., Citrus Research and Education Center, Lake Alfred, FL,

U.S.A.

Green, S., Forest Research,, Roslin, United Kingdom

Grewal, T., Saskatchewan Research Council, Saskatoon,

Saskatchewan, Canada

Gross, D., Texas A\&M University, College Station, TX, U.S.A.

Grove, G. G., Washington State University, Prosser, WA, U.S.A.

Grunwald, N. J., USDA-ARS, Corvallis, OR, U.S.A.

Gudmestad, N. C., North Dakota State University, Fargo, ND, U.S.A.

Hajimorad, R., University of Tennessee, Knoxville, TN, U.S.A.

Halbrendt, J., Penn State, Biglerville, PA, U.S.A.

Halterman, D., USDA-ARS, Madison, WI, U.S.A.

Hammerschmidt, R., Michigan State University, East Lansing, MI,

U.S.A.

Hammond, J., U.S. Department of Agriculture

Harman, G., Cornell University, Geneva, NY, U.S.A.

Harp, T., Syngenta Crop Protection, Greensboro, NC, U.S.A.

Harrington, T., Iowa State University, Ames, IA, U.S.A.

Harrison, N., University of Florida, Fort Lauderdale, FL, U.S.A.

Hartman, G., USDA-ARS and University of Illinois, Urbana, IL,

U.S.A.

Hartung, J. S., USDA-ARS MPPL, Beltsville, MD, U.S.A.

Hau, B. E., University of Hannover, Hannover, Germany

Haydock, P., Harper Adams University College, Newport, United

Kingdom

Hebrard, E., IRD, Montpellier, France

Heesterbeek, $\mathrm{H}$.

Helder, J., Wageningen University, Wageningen, Netherlands

Hewezi, T., Iowa State University, Ames, IA, U.S.A.

Holb, I. J., University of Debrecen, Debrecen, Hungary

Howard, R., DuPont Co, Wilmington, DE, U.S.A.

Huang, B.

Huber, D. M., Purdue University, West Lafayette, IN, U.S.A.

Huckelhoven, R., Technische Universitaet Muenchen, Freising,

Germany

Hughes, T., University of Wisconsin, Madison, WI, U.S.A.

Hussey, R. S., University of Georgia, Athens, GA, U.S.A.

Ibekwe, A. M., USDA-ARS, Riverside, CA, U.S.A.

Inderbitzin, P., University of California, Davis, CA, U.S.A.

Ingram, D., Mississippi State University, Raymond, MS, U.S.A.

Isakeit, T., College Station, TX, U.S.A.

Isard, S., Pennsylvania State University, University Park, PA, U.S.A.

Ito, Y., National Food Research Institute, Tsukuba, Ibaraki, Japan

James, D., Centre for Plant Health, Sidney, British Columbia, Canada

Janda, T., Agricultural Research Institute of the Hungarian Academy

of Sciences, Martonvásár, Hungary

Jansky, S. H., University of Wisconsin, Madison, WI, U.S.A.
Jarosz, A. M., Michigan State University, East Lansing, MI, U.S.A. Jeger, M. J., Imperial College London, Ashford, United Kingdom

Jena, K. K., International Rice Research Institute, Makati City, Philippines

Jenkins, D. M., University of Hawaii, Honolulu, HI, U.S.A.

Jia, Y., USDA-ARS, Stuttgart, AR, U.S.A.

Jiménez-Díaz, R. M., Instituto Agricultura Sostenible, Córdoba, Spain

Jin, Y., USDA-ARS Cereal Disease Laboratory, St. Paul, MN, U.S.A.

Jo, Y., Texas A\&M University, College Station, TX, U.S.A.

Johnson, D. A., Washington State University, Pullman, WA, U.S.A.

Johnson, K., Department of Botany and Plant Pathology, Corvallis, U.S.A.

Joly, D. L., Agriculture and Agri-Food Canada, Summerland, British Columbia, Canada

Jones, J. B., University of Florida, Gainesville, FL, U.S.A.

Jurick, W. M., II, USDA-ARS, Beltsville, MD, U.S.A.

Juvale, P., Iowa State University, Ames, IA, U.S.A.

Kaloshian, I., University of California, Riverside, CA, U.S.A.

Kang, S., Penn State, University Park, PA, U.S.A.

Kanter, D., Mississippi, Stoneville, MS, U.S.A.

Kanyuka, K., Rothamsted Research, Harpenden, United Kingdom

Karasev, A., University of Idaho, Moscow, ID, U.S.A.

Kassemeyer, H., Staatliches Weinbauinstitut, Freiburg im Breisgau, Germany

Kasson, M.

Katan, J., Hebrew University, Rehovot, Israel

Keinath, A., Clemson University, Charleston, SC, U.S.A.

Kelly, J., Michigan State Univ., East Lansing, MI, U.S.A.

Kikuchi, T., Forestry and Forest Product Research Institute, Tsukuba, Ibaraki, Japan

Killiny, N., University of Florida, Lake Alfred, FL, U.S.A.

Kinkel, L. L., University of Minnesota, St. Paul, MN, U.S.A.

Kirkpatrick, B., University of California, Davis, CA, U.S.A.

Kläring, H.

Klosterman, S. J., USDA-ARS, Salinas, CA, U.S.A.

Kluepfel, D., USDA-ARS, Davis, CA, U.S.A.

Koebnik, R., Institut de Recherche pour le Développement, Montpellier, France

Koenning, S., North Carolina State University, Raleigh, NC, U.S.A.

Kolmer, J., USDA-ARS, St. Paul, MN, U.S.A.

Krattinger, D., CSIRO Plant Industry, Canberra, Australian Capital Territory, Australia

Krenz, B.

Kriss 2, A., USDA-ARS, Fort Pierce, FL, U.S.A.

Kunjeti, S., University of Delaware, Newark, DE, U.S.A.

Kushalappa, A. C., McGill University, Ste. Anne de Bellevue, Quebec, Canada

LaMondia, J., The Connecticut Agricultural Experiment Station, Windsor, CT, U.S.A.

Landa, B. B., Institute for Sustainable Agriculture-CSIC, Cordoba, Spain

Larkin, P.

Larkin, R., USDA-ARS, Orono, ME, U.S.A.

Lazarovits, G., A\&L Biologicals, London, Ontario, Canada

Lazzeri, L., CRA, Bologna, Italy

Leandro, L., Iowa State University, Ames, IA, U.S.A.

Lee, R. F., USDA-ARS, Riverside, CA, U.S.A.

Lescourret, F., INRA, Avignon cedex 9, France

Leslie, J. F., Kansas State University, Manhattan, KS, U.S.A.

Lett, J., CIRAD, Saint-Pierre, Ile de la Réunion, France

Leveau, J., University of California, Davis, CA, U.S.A.

Levine, A., The Hebrew University of Jerusalem, Jerusalem, Israel

Levy, L. E., USDA-APHIS PPQ-CPHST National Plant Germplasm and Biotechnology Laboratory, Beltsville, MD, U.S.A.

Li, S., Canadian Food Inspection Agency, Charlottetown, Prince

Edward Island, Canada

Li, W., USDA-APHIS, Beltsville, MD, U.S.A.

Lindeberg, M., Cornell University, Ithaca, NY, U.S.A.

Little, C. R., Kansas State University, Manhattan, KS, U.S.A. 
Liu, S., Texas A\&M University, Amarillo, TX, U.S.A.

Loper, J., USDA-ARS, Corvallis, OR, U.S.A.

Lopes, C. A., Embrapa, Brasilia, DF, Brazil

Lopes, J. S., ESALQ/Universidade de São Paulo, Piracicaba, SP, Brazil

Lopes, S., Fundecitrus, Araraquara, São Paulo, Brazil

Lu, S., Mississippi State University, Mississippi State, U.S.A.

Lübeck, M., Aalborg University, Ballerup, Denmark

Luster, D., USDA-ARS, Ft. Detrick, MD, U.S.A.

Madden, L. V., Ohio State University, Wooster, OH, U.S.A.

Magarey, R., Raleigh, NC, U.S.A.

Magill, C., Texas A\&M, College Station, TX, U.S.A.

Mansoor, $\mathrm{S}$.

Marco, M., University of California, Davis, CA, U.S.A.

Marra, R. E., Connecticut Agricultural Experiment Station, New

Haven, CT, U.S.A.

Martin, F. N., USDA-ARS, Salinas, CA, U.S.A.

Martyn, R., Purdue University, West Lafayette, IN, U.S.A.

Maruthachalam, K., University of California-Davis, Salinas, CA, U.S.A.

Mastouri, F., Cornell University, Geneva, NY, U.S.A.

Mathieu, J., Boyce Thompson Institute, Ithaca, NY, U.S.A.

Maule, A., Queen's University Belfast, Belfast, United Kingdom

Mavrodi, D., Washington State University, Pullman, WA, U.S.A.

Mazzola, M., USDA-ARS, Wenatchee, WA, U.S.A.

McCallum, B., Agriculture and Agri-Food Canada, Winnipeg,

Manitoba, Canada

McGrath, M., Cornell University, Riverhead, NY, U.S.A.

McGrath, M., Fitchburg, WI, U.S.A.

McRoberts, N., UC Davis, Davis, CA, U.S.A.

McSpadden Gardener, B., Wooster, OH, U.S.A.

Mehl, H. L., USDA-ARS, University of Arizona, Tucson, AZ, U.S.A.

Melotto, M., University of Texas, Arlington, TX, U.S.A.

Mengiste, T., Purdue University, West Lafayette, IN, U.S.A.

Menzel, W., Leibniz Institute DSMZ, Braunschweig, Germany

Metz, H., Rotterdam, Netherlands

Mideros, S. X., Cornell University, Ithaca, NY, U.S.A.

Mila, A., North Carolina State University, Raleigh, NC, U.S.A.

Miller, S. A., The Ohio State University, Wooster, OH, U.S.A.

Millner, P., USDA-ARS, Beltsville, MD, U.S.A.

Milus, G., University of Arkansas, Fayetteville, AR, U.S.A.

Miyasaka, S. C., University of Hawaii, Hilo, HI, U.S.A.

Moreno, P., Valenciano de Investigaciones Agrarias (IVIA),

Moncada (Valencia), Valencia (SPAIN), Spain

Mundt, C., Oregon State University, Corvallis, OR, U.S.A.

Munkvold, G., Iowa State University, Ames, IA, U.S.A.

Murray, T., Washington State University, Pullman, WA, U.S.A.

Mysore, K., Ardmore, OK, U.S.A.

Naidu, R., Washington State University, Prosser, WA, U.S.A.

Nault, B., NYSAES, Geneva, NY, U.S.A.

Nelson, E. B., Cornell University, Ithaca, NY, U.S.A.

Nelson, R. S., Samuel Roberts Noble Foundation, Inc., Ardmore, OK, U.S.A.

Neri, F., University of Cambridge, Cambridge, United Kingdom

Ngugi, H. K., Penn State University, Biglerville, PA, U.S.A.

Nicole, M., IRD, Montpellier, France

Nicot, P., INRA, Montfavet, France

Nie, X., Potato Research Centre, Fredericton, New Brunswick, Canada

Nita, M., Virginia Tech, Winchester, VA, U.S.A.

Nolasco, G., Universidade do Algarve, Faro, Portugal

Norelli, J. L., USDA-ARS, Kearneysville, WV, U.S.A.

Nyczepir, A. P., USDA-ARS, Byron, GA, U.S.A.

Ojiambo, P. S., North Carolina State University, Raleigh, NC, U.S.A.

Olanya, J., University of Hawaii, Honolulu, HI, U.S.A.

Oliver, J., Auburn University, Auburn, AL, U.S.A.

Oliver, R. P., Murdoch University, Perth, Western Australia,

Australia

Olukolu, B., North Carolina State University, Raleigh, NC, U.S.A.

Pachepsky, Y., USDA-ARS, Beltsville, MD, U.S.A.

Palomares Rius, J., Scottish Crop Research Institute-Invergowrie,

Invergowrie, Dundee, Scotland, United Kingdom
Palumbo, J. D., USDA-ARS, Albany, CA, U.S.A.

Panabières, F., INRA, France

Pappu, H., Washington State University, Pullman, WA, U.S.A.

Pardyjak, E., University of Utah, Salt Lake City, UT, U.S.A.

Paret, M. L., NFREC, University of Florida, Quincy, FL, U.S.A.

Parnell, S., Rothamsted Research, Harpenden, Hertfordshire, United Kingdom

Pattemore, J. A., Charles Sturt University, North Wagga, New South Wales, Australia

Paul, P. A., Ohio State University, Wooster, OH, U.S.A.

Paulitz, T. C., USDA-ARS, Pullman, WA, U.S.A.

Pedley, K. F., USDA-ARS, Ft. Detrick, MD, U.S.A.

Pereira, F. V., EMBRAPA Agricultural Instrumentation, São Carlos, São Paulo, Brazil

Peres, N., University of Florida, Wimauma, FL, U.S.A.

Perry, K. L., Cornell University, Ithaca, NY, U.S.A.

Pethybridge, S. J., Botanical Resources Australia-Agricultural

Services Pty. Ltd., Ulverstone, Tasmania, Australia

Pfender, W., Corvallis, OR, U.S.A.

Pierson, E. A., Texas A\&M University, College Station, TX, U.S.A.

Pietravalle, S., Fera, York, United Kingdom

Pinkard, L., CSIRO, Hobart, Victoria, Australia

Pirhonen, $\mathrm{M}$.

Pitman, A. R., The New Zealand Institute for Plant \& Food Research, Christchurch, New Zealand

Ploetz, R., Tropical Research and Education Center, University of

Florida, Homestead, FL, U.S.A.

Poland, J., USDA-ARS, Manhattan, KS, U.S.A.

Ponder, $\mathrm{M}$.

Powers, T. O., University of Nebraska, Lincoln, NE, U.S.A.

Prince, J.

Prior, P., CIRAD-INRA, Saint Pierre, La Réunion, Réunion

Prusky, D., Volcani Center, Rishon Le Tzion, Israel

Pryor, B., Tucson, AZ, U.S.A.

Punja, Z. K., Simon Fraser University, Burnaby, British Columbia, Canada

Qu, F., Ohio State University, Wooster, OH, U.S.A.

Qu, X., Pennsylvania State University, University Park, PA, U.S.A.

Quesda-Ocampo, L., East Lansing, MI, U.S.A.

Rabenstein, F., JKI, Quedlinburg, Germany

Rahman, M., West Virginia University, Morgantown, WV, U.S.A.

Raposo, R., INIA, Madrid, Spain

Rashed, A., Texas A\&M AgriLife Research, Amarillo, TX, U.S.A.

Restrepo, S., Universidad de los Andes, Bogotá, Colombia

Reverberi, M., Università Sapienza, Roma, Italy

Rideout, $\mathrm{S}$.

Ristaino, J. B., North Carolina State University, Raleigh, NC, U.S.A.

Ritchie, D., North Carolina State University, Raleigh, NC, U.S.A.

Rizzo, D. M., Univ of California, Davis, CA, U.S.A.

Roberts, P. A., University of California, Riverside, CA, U.S.A.

Robertson, A., Iowa State University, Ames, IA, U.S.A.

Rohrs-Richey, J.

Rollins, J. A., University of Florida, Gainesville, FL, U.S.A.

Rosenberger, D., Cornell University/NYAES, Highland, NY, U.S.A.

Rossi, V., Università Cattolica del Sacro Cuore, Piacenza, Italy

Rouse, M. N., USDA-ARS Cereal Disease Laboratory, St. Paul, MN, U.S.A.

Ruiz-Herrera, J., CINVESTAV del IPN, Irapuato, Mexico

Runge, F., University of Hohenheim, Stuttgart, Germany

Rupe, J. C., University of Arkansas, Fayetteville, AR, U.S.A.

Sabanadzovic, S., Mississippi State University, MS, U.S.A.

Sache, I., INRA, Thiverval-Grignon, France

Sanogo, S., New Mexico State University, Las Cruces, NM, U.S.A.

Saponari, M., Istituto di Virologia Vegetale CNR UOS di Bari, Bari, Italy

Savary, S., INRA, Castanet Tolosan, France

Scheets, K., Oklahoma State University, Stillwater, OK, U.S.A.

Schepers, H., Applied Plant research, Lelystad, Flevoland, Netherlands

Scherm, H., University of Georgia, Athens, GA, U.S.A.

Schirawski, J., Georg-August-University, Göttingen, Germany 
Schisler, D.

Schmale, D., Virginia Polytechnic Institute and State University,

Blacksburg, VA, U.S.A.

Schnabel, G., Clemson University, Clemson, SC, U.S.A.

Schneider, R. W., Louisiana State University, Baton Rouge, LA,

U.S.A.

Schoelz, J. E., University of Missouri, Columbia, MO, U.S.A.

Scholthof, H., College Station, U.S.A.

Schroeder, B., Washington State University, Pullman, WA, U.S.A.

Schroeder, K., Washington State University, Pullman, WA, U.S.A.

Scofield, S., USDA-ARS, West Lafayette, IN, U.S.A.

Scortichini, M., CRA-Istituto Sperimentale per la Frutticoltura,

Roma, Italy

Scott, I., NZ Institute for Plant and Food Research, Christchurch,

New Zealand

Scott, S., Clemson University, Clemson, SC, U.S.A.

Seebold, K. W., University of Kentucky, Lexington, KY, U.S.A.

Segarra, J., Universitat de Lleida, Lleida, Spain

Semenov, M., RREs, Harpenden, United Kingdom

Sha, X.

Shaw, M., University of Reading, Reading, United Kingdom

Shew, D., North Carolina State University, Raleigh, NC, U.S.A.

Shiel, P., U.S. Department of Agriculture, Raleigh, NC, U.S.A.

Shim, W., Texas A\&M University, College Station, TX, U.S.A.

Simko, I., USDA-ARS, Salinas, CA, U.S.A.

Singh, K.

Skaria, M., Texas A\&M University-Kingsville, Weslaco, TX, U.S.A.

SkŁodowska, M., Lodz, Poland

Smant, G., Wageningen University, Wageningen, Netherlands

Smart, C. D., Cornell University, Geneva, NY, U.S.A.

Smith, B. J., USDA-ARS Southern Horticultural Laboratory,

Poplarville, MS, U.S.A.

Smith, M., Texas AgriLife Research, Amarillo, TX, U.S.A.

Smith, S. M., University of Georgia, Athens, GA, U.S.A.

Solomon, P., The Australian National University, Canberra,

Australian Capital Territory, Australia

Sonti, R.

Spotts, R., Hood River, OR, U.S.A.

Stenger, D. C., USDA-ARS, Parlier, CA, U.S.A.

Stewart, L.

Stoll, R.

Stone, J. K., Oregon State University, Corvallis, OR, U.S.A.

Subbotin, S., CA Department of Food and Agriculture, Sacramento,

CA, U.S.A.

Sudarshana, M., University of California, Davis, CA, U.S.A.

Sunter, G., University of Texas at San Antonio, San Antonio, TX,

U.S.A.

Suslow, T., University of California, Davis, CA, U.S.A.

Sutton, T. B., North Carolina State University, Raleigh, NC, U.S.A.

Svircev, A. M., Agriculture and Agri-Food Canada, Vineland Station,

Ontario, Canada

Takamatsu, S., Mie University, Tsu, Mie, Japan

Talhinhas, P., Instituto de Investigação Científica Tropical, Oeiras, Portugal

Tegli, S., Università di Firenze, Sesto Fiorentino, Italy

Tennant, P., University of the West Indies, Kingston, Jamaica
Thilmony, R.

Thines, $\mathrm{M}$.

Thomashow, L., USDA-ARS, Pullman, WA, U.S.A.

Tian, $\mathrm{P}$.

Toth, I., The James Hutton Institute

Toussaint, V., Agriculture and Agri-Food Canada, St-Jean-sur-

Richlieu, Quebec, Canada

Trillas, M., Universidad de Barcelona, Barcelona, Spain

Triplett, L., Colorado State University, Fort Collins, CO, U.S.A.

Turechek, W. W., USDA-ARS USHRL, Fort Pierce, FL, U.S.A.

Tzanetakis, I., University of Arkansas, Fayetteville, AR, U.S.A.

Upchurch, G., USDA-ARS, Raleigh, NC, U.S.A.

Uphaus, J., DuPont Pioneer, Windfall, IN, U.S.A.

Vaillancourt, L. J., University of Kentucky, Lexington, KY, U.S.A.

Vallad, G. E., University of Florida, Wimauma, FL, U.S.A.

van Bruggen, A. H., University of Florida, Gainesville, FL, U.S.A.

van der Werf, W., Wageningen University, Wageningen, Netherlands

Varsani, A., University of Canterbury, Christchurch, New Zealand

Veronese, P., NCSU, Raleigh, NC, U.S.A.

Vidalakis, G., University of California, Riverside, CA, U.S.A.

Viljoen, A., Stellenbosch University, Stellenbosch, Western Cape, South Africa

Vinatzer, B., Virginia Tech, Blacksburg, VA, U.S.A.

Voegele, R. T., Universität Hohenheim, Stuttgart, Germany

Vorholt, J.

Wang, J., AVRDC-The World Vegetable Center, Shanhua, Tainan, Taiwan

Wang, G., North Carolina State University, Raleigh, NC, U.S.A.

Wang, G., Ohio State University, Columbus, OH, U.S.A.

Wang, N., University of Florida, Lake Alfred, FL, U.S.A.

Warburton, M., USDA, U.S.A.

Wei, T.

Welham, S., Rothamsted Research, Harpenden, Herts, United Kingdom

West, J., Rothamsted Research, Harpenden, United Kingdom

Westphal, A., Julius Kühn-Institut, Münster, Germany

Wheeler, T., Texas AgriLife Research, Lubbock, TX, U.S.A.

White, F., Manhattan, KS, U.S.A.

White, J.

Whitfield, A., Kansas State University, Manhattan, KS, U.S.A.

Whitham, S., Iowa State University, Ames, IA, U.S.A.

Wicklow, D., NCAUR-ARS-USDA, Peoria, IL, U.S.A.

Willocquet, L., International Rice Research Institute (IRRI), Metro Manila, Philippines

Wise, K., Purdue University, West Lafayette, IN, U.S.A.

Wisser, R. J., University of Delaware, Newark, DE, U.S.A.

Wu, B., Oregon State University, Madras, OR, U.S.A.

Wubben, M., USDA-ARS, Mississippi State, MS, U.S.A.

Xiao, C., Washington State University, Wenatchee, WA, U.S.A.

Yan, Q., University of Florida, Lake Alfred, FL, U.S.A.

Yeh, H., National Taiwan University, Taipei, Taiwan

Yeh, S., National Chung Hsing University, Taichung City, Taiwan

Yoder, K., Virginia Tech AREC, Winchester, VA, U.S.A.

Yuen, J. E., Swedish University of Agriculture Science, Uppsala, Sweden

Zechmann, B., University of Graz, Graz, Austria

Zhou, S., Texas A\&M University System, Beaumont, TX, U.S.A. 